\title{
Cenizas de calderas dendroenergéticas. I Acción como enmienda alcalina en suelos ácidos de la zona sur de Chile
}

\author{
A shes of wood-burning ovens. I Effects of using wood Ash as an \\ alkaline amendmer in acid soils of southern Chile
}

C.D.0.: $114.25,232.322 .49$

RENATO GREZ, VICTOR GERDING Y FRANCISCO UNION

Instituto de Silvicultura, Universidad A ustral de Chile, Casilla 567, Valdivia, Chile.

\section{SUMMARY}

Wood combustion has become an energy source of growing importance for Chile. As a byproduct, wood ash offers a growing potential resource for use as a soil amendmer. A shes are inorganic, mostly composed of mineral elements present in the wood tissue, such as $\mathrm{K}, \mathrm{Ca}, \mathrm{M} \mathrm{g}$, or others. These oxidized elements hydrolize with water to form alkaline hydroxides. This effect, when wood ash is applied to acid soils as an amendmer, raises pH one or more units depending upon the quantity of ash added. As a result, use of wood ash as a soil amendmer is valuable to regulate soil $\mathrm{pH}$ and promote plant growth.

\section{RESUMEN}

La combustión de recursos leñosos ha pasado a constituir una fuente de abastecimiento energético de creciente importancia para Chile. Como resultado de dicha combustión quedan las cenizas, producto de carácter inorgánico constituido por la mayor parte de los elementos minerales presentes en el tejido leñoso, tales como K, $\mathrm{Ca}$ y M g, entre otros. Estos elementos, que se encuentran en forma de óxidos, sufren una hidrólisis al reaccionar con agua, transformándose en hidróxidos de carácter alcalino. Tal efecto, al ser combinado con suelos ácidos, conduce a un incremento del pH en rangos variables de una o más unidades, dependiendo de la cantidad de ceniza adicionada, $y$, por lo tanto, constituye un valioso mecanismo para regular este parámetro del suelo que en gran medida condiciona la adaptación y la producción de especies vegetales.

\section{INTRODUCCION}

La dendroenergía, o energía proveniente de la combustión de materiales leñosos, es una fuente energética de creciente demanda en el país, debido a su valor competitivo con otros tipos de combustibles. En Chile, aproximadamente el $25 \%$ de la energía requerida por la población se obtiene de leña y de otros residuos de la industria maderera. A sí, entre julio de 1987 y junio de 1988 tan sólo entre Talca y Temuco se utilizaron 1.160 .000 toneladas de aserrín para dicho propósito (Chile Forestal, 1987a, b y c). Basándose en estas cifras se puede estimar que de tal combustión se produjo entre $40 \mathrm{mil}$ a $50 \mathrm{mil}$ toneladas de cenizas.
El interés por buscar una alternativa de uso para este residuo condujo al desarrollo del presente estudio, cuyo objetivo comprende tanto aspectos de caracterización de las cenizas, en cuanto a su composición química, así como de su reactividad con el suelo. Su ejecución fue posible gracias al financiamiento del Fondo Nacional de Ciencia y Tecnología, FONDECY T*.

Los resultados correspondientes se presentan en una serie de 4 publicaciones, abarcando los tópicos principales:

- Cenizas de calderas dendroenergéticas. I Acción como enmienda alcalina en suelos ácidos de la zona sur de Chile.

* Proyecto 89/0199 financiado por el Fondo Nacional de Ciencia y Tecnología (FONDECYT). 
- Cenizas de calderas dendroenergéticas. II Residuo industrial utilizable como mejorador de la fertilidad del suelo.

- Cenizas de calderas dendroenergéticas. III Efecto amortiguador sobre la fijación de fósforo en suelos ácidos con aluminio activo.

- Cenizas de calderas dendroenergéticas. IV Efecto sobre la absorción de elementos nutritivos en vegetales.

\section{MATERIAL Y METODO}

Cenizas. Se seleccionaron cenizas provenientes de calderas dendroenergéticas de cinco industrias de la ciudad de $V$ aldivia, que representan distintos tipos de combustibles leñosos, según se detalla en el cuadro 1.

Estas cenizas, recogidas durante la limpieza de los hornos de combustión, mediante un muestreo sistemático, fueron analizadas por simplificado, en cuanto a su pH y a la solubilización de elementos $\mathrm{P}, \mathrm{K}, \mathrm{Ca}$ y $\mathrm{Mg}$ en agua destilada. En base a dichos resultados se procedió a seleccionar una ceniza representativa para la serie de ensayos posteriores. Dada esta gran variabilidad encontrada se decidió elegir una ceniza que representara valores intermedios y que a su vez su origen fuese del tipo de material que constituye una fuente principal de suministro de combustible. En tal sentido se seleccionó para las siguientes fases del estudio la ceniza CEFOR.

\section{CUADRO 1}

Combustible utilizado en calderas dendroenergéticas de cinco industrias de Valdivia

Fuel used in five wood-burning ovens of industries of Valdivia

\begin{tabular}{|c|c|}
\hline Industria & Tipo de combustible \\
\hline INFODEMA & $\begin{array}{l}\text { Leña de maderas nativas (tepa. } \\
\text { roble, otras) }\end{array}$ \\
\hline CEFOR & $\begin{array}{l}\text { Corteza, madera, residuos varios } \\
\text { de pino insigne }\end{array}$ \\
\hline EMASIL & $\begin{array}{l}\text { Viruta-aserrín de maderas nati- } \\
\text { vas y de pino insigne }\end{array}$ \\
\hline COLLICO & $\begin{array}{l}\text { Mezcla de maderas nativas y de } \\
\text { eucalipto }\end{array}$ \\
\hline MASISA A & $\begin{array}{l}\text { Astillas de maderas nativas y de } \\
\text { pino insigne }\end{array}$ \\
\hline MASISA B & Astillas de maderas nativas \\
\hline
\end{tabular}

Suelos. Se seleccionaron tres tipos de suelos representativos de la zona sur del país: a) trumao, derivados de materiales piroclásticos, serie $V$ aldivia, recolectado en la ciudad de Valdivia; b) rojo arcilloso, derivados de materiales piroclásticos, serie $M$ etrenco, obtenido en el sector Padre de las Casas en la provincia de Cautín, y c) pardo metamórfico, derivado de rocas micaesquísticas, asociación Tres Cruces, fue muestreado en el sector costero de la provincia de $\mathrm{V}$ aldivia, entre $\mathrm{Niebla}$ y Curiñanco (IREN-CORFO-UACH，1978).

Caracterización de mezcla de cenizas y suelos. La ceniza fue mezclada en proporciones de 0.5 , $1.0,2.5,4.0,7.0$ y $10.0 \%$ en relación peso/peso con cada suelo, determinándose en dichas mezclas la variación del $\mathrm{pH}$. En base a estos resultados se seleccionaron tres porcentajes de ceniza, adecuados para elevar el pH natural de los suelos a un rango comprendido entre 6 y 7, ya que en él la mayoría de los vegetales puede adaptarse sin dificultades y además las reacciones químicas de solubilidad que ocurren en el suelo no se ven restringidas severamente (A dams, 1984; Bolt, B ruggenwert, 1978; Finck, 1979). Con estas proporciones se preparó una cantidad suficiente para completar el volumen de 4 maceteros de 5 litros por cada mezcla. En ellos se verificó la variación del pH en el tiempo.

Determinaciones. $L$ as determinaciones antes señaladas se efectuaron según el siguiente procedimiento:

- Solubilización de P, K, Ca y M g en agua: $1 \mathrm{~g}$ de ceniza se mezcló con $100 \mathrm{mi}$ de agua desionizada y se agitó durante 2 horas en agitador rotatorio. Luego de 18 horas de reposo se repitió la agitación por 1 hora y se filtró, precediéndose al análisis del filtrado. El pH fue medido electrométricamente por intermedio de un electrodo doble (Schlichting, Blume, 1966), y los elementos $\mathrm{K}, \mathrm{Ca}$ y $\mathrm{Mg}$ fueron determinados por espectrofotometría de absorción atómica (Perkin Elmer, 1982). P fue determinado colométricamente por el método de Murphy, Riley (1962).

- Reacción o pH de mezclas suelo-ceniza:

a) Efecto según dosis. La acción de las diferentes dosis fue estudiada haciendo reaccionar $10 \mathrm{~g}$ de la mezcla con $25 \mathrm{ml}$ de agua desionizada, agitando intermitentemente por dos horas y midiendo el $\mathrm{pH}$ en el sobrenadante luego de un reposo de media hora. 
b) Efecto de la adición de cenizas en el tiempo. La mezcla preparada en maceteros, que se mantuvo a la intemperie durante todo el período del ensayo, fue controlada periódicamente en cuanto a su variación de $\mathrm{pH}$. Para ello se preparó una muestra representativa de los 4 maceteros de cada dosis $y$, en forma idéntica a la señalada anteriormente, se procedió a determinar su $\mathrm{pH}$. Las mediciones fueron realizadas al momento de la mezcla y a los $7,14,26,41$ y 328 días.

\section{RESULTADOS Y DISCUSION}

De acuerdo a los resultados de caracterización química de las cenizas en cuanto a su pH y a la solubilización de $\mathrm{P}, \mathrm{K}, \mathrm{Ca}$ y $\mathrm{Mg}$ en agua desionizada (cuadro 2) se puede apreciar que la mayoría presenta una reacción fuertemente alcalina, derivada de su carácter químico de óxidos, los cuales, en contacto con agua, sufren una hidrólisis, liberando grupos $\mathrm{OH}$ - y superando así valores de $\mathrm{pH} 11$ y 12. Respecto a los niveles de elementos nutritivos, éstos alcanzan cifras muy variables.

\section{CUADRO 2}

Solubilización de algunos elementos nutritivos y $\mathbf{p H}$ en agua de cenizas de calderas dendroenergéticas Concentrations of $\mathrm{pH}$ and selected elements in water of ashes from various wood-burning ovens

\begin{tabular}{|c|c|c|c|c|c|}
\hline \multirow[b]{2}{*}{ CENIZA } & \multirow[b]{2}{*}{ pH Agua } & \multicolumn{4}{|c|}{$\mathrm{mg} / 100 \mathrm{~g}$ ceniza seca a $105^{\circ} \mathrm{C}$} \\
\hline & & $\mathbf{P}$ & $\mathbf{K}$ & $\mathbf{C a}$ & Mg \\
\hline INFODEMA & 12.1 & 7 & 3981 & 80 & 27 \\
\hline CEFOR & 11.3 & 16 & 1053 & 201 & $<1$ \\
\hline EMASIL & 11.0 & 42 & 3609 & 78 & 70 \\
\hline COLLICO & 12.9 & $<1$ & 10674 & 36 & $<1$ \\
\hline MASISA A & 12.3 & 1 & 5751 & 10 & $<1$ \\
\hline MASISA B & 10.2 & 3 & 209 & 328 & 98 \\
\hline
\end{tabular}

\section{CUADRO 3}

Reacción (pH) de diferentes mezclas suelo-ceniza

pH Change of different soil-ash mixtures over time

\begin{tabular}{|c|c|c|c|c|c|c|}
\hline Suelo & $\%$ Ceniza & Día 1 & Día 2 & Día3 & Día 4 & Día 5 \\
\hline \multirow{7}{*}{$\begin{array}{l}\text { Rojo } \\
\text { arcilloso }\end{array}$} & 0.0 & 5.05 & 5.09 & 5.10 & 5.12 & 5.14 \\
\hline & 0.5 & 6.35 & 6.25 & 6.15 & 6.09 & nd * \\
\hline & 1.0 & 6.45 & 6.52 & 6.53 & 6.49 & 6.48 \\
\hline & 2.5 & 7.41 & 7.48 & 7.38 & 7.31 & nd \\
\hline & 4.0 & 7.60 & 7.73 & 7.73 & 7.69 & 7.72 \\
\hline & 7.0 & 8.10 & 8.15 & 8.23 & 8.28 & 8.33 \\
\hline & 10.0 & 8.50 & 8.52 & 8.62 & 8.72 & 8.83 \\
\hline \multirow{7}{*}{$\begin{array}{l}\text { Meta- } \\
\text { mórfico }\end{array}$} & 0.0 & 5.45 & 5.49 & 5.50 & 5.58 & 5.58 \\
\hline & 0.5 & 6.15 & 6.18 & 6.13 & 6.13 & nd \\
\hline & 1.0 & 6.55 & 6.51 & 6.52 & 6.55 & 6.54 \\
\hline & 2.5 & 7.05 & 7.05 & 7.06 & 7.07 & nd \\
\hline & 4.0 & 7.43 & 7.50 & 7.52 & 7.52 & 7.59 \\
\hline & 7.0 & 7.70 & 7.77 & 7.84 & 7.87 & 7.92 \\
\hline & 10.0 & 8.10 & 8.13 & 8.20 & 8.26 & 8.35 \\
\hline \multirow{7}{*}{ Trumao } & 0.0 & 5.38 & 5.40 & 5.40 & 5.43 & 5.41 \\
\hline & 0.5 & 6.07 & 6.10 & 6.07 & 6.02 & nd \\
\hline & 1.0 & 6.40 & 6.35 & 6.35 & 6.35 & 6.40 \\
\hline & 2.5 & 7.07 & 7.11 & 7.05 & 7.03 & nd \\
\hline & 4.0 & 7.11 & 7.15 & 7.20 & 7.22 & 7.25 \\
\hline & 7.0 & 7.35 & 7.55 & 7.63 & 7.63 & 7.65 \\
\hline & 10.0 & 7.82 & 7.91 & 7.99 & 7.99 & 8.05 \\
\hline
\end{tabular}

* nd: no determinado 
SUELO ROJO ARCILLOSO (a)

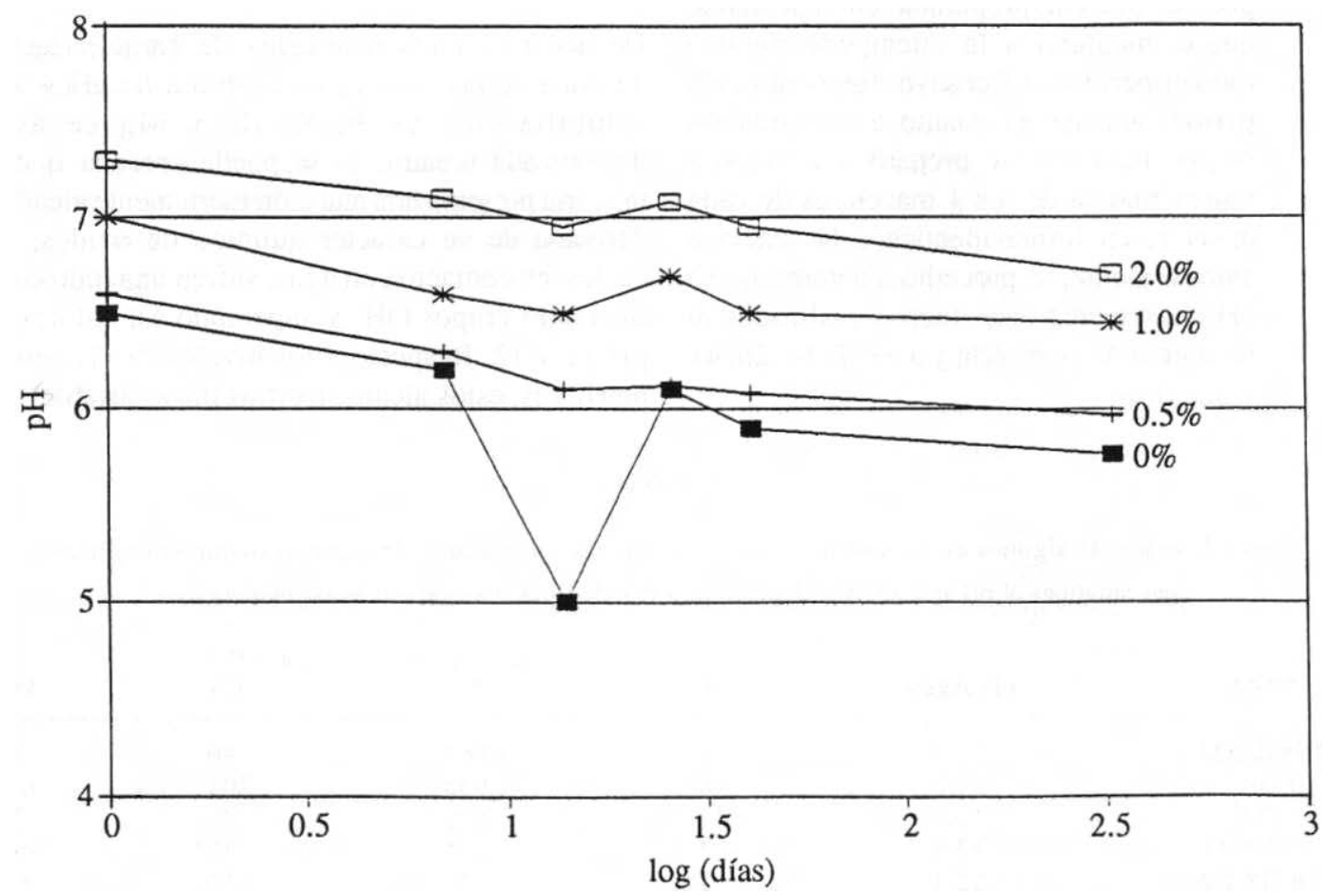

SUELO PARDO METAMORFICO (b)

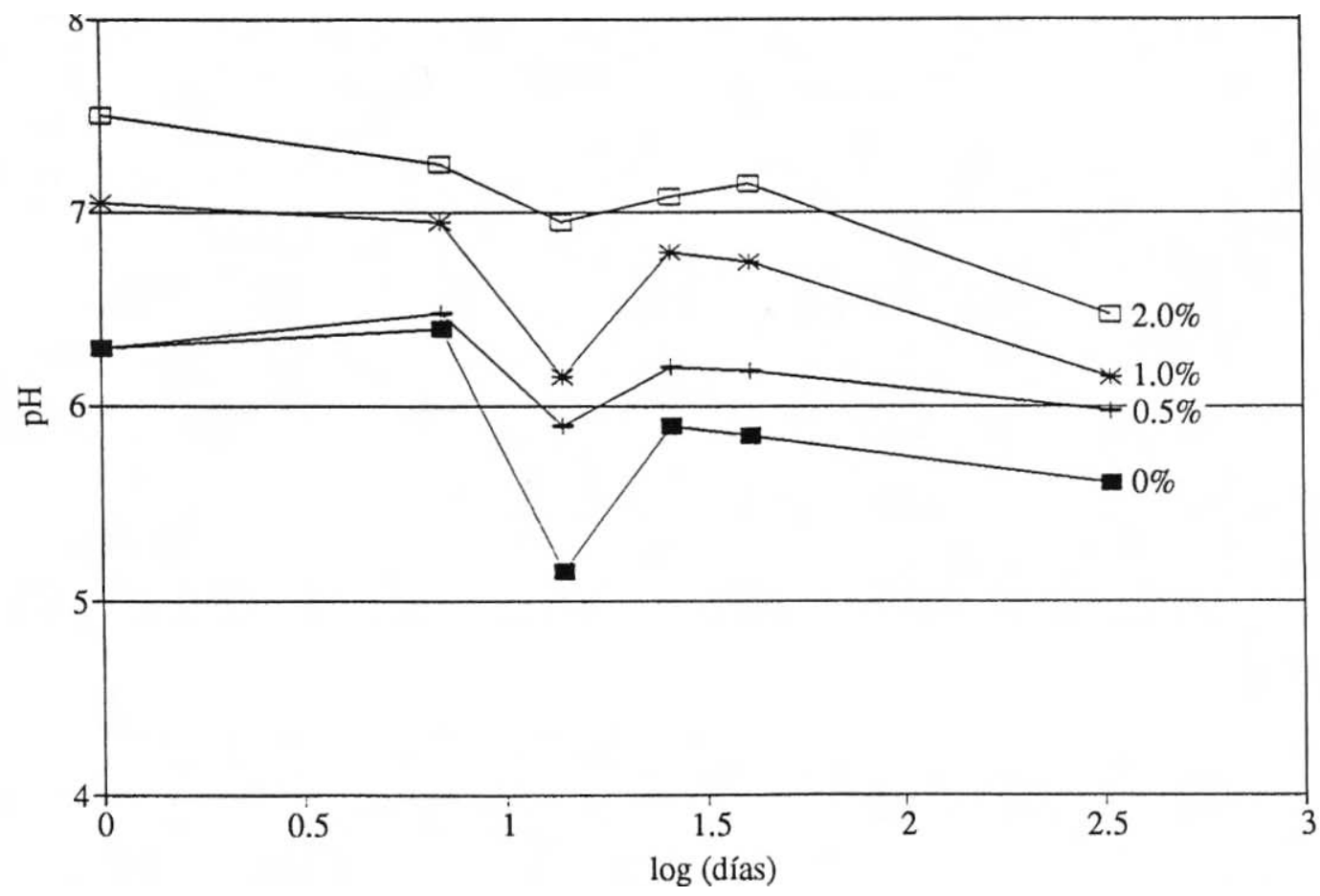

Fig. 1. Variación del pH en el tiempo para mezclas de suelos rojo arcilloso (a) y metamórfico (b) con ceniza en distintas dosis.

$\mathrm{pH}$ variation over time for mixtures of 'red clay' (a), 'metamorphic' (b) soils with wood ash of distinct doses. 
SUELO TRUMAO (c)

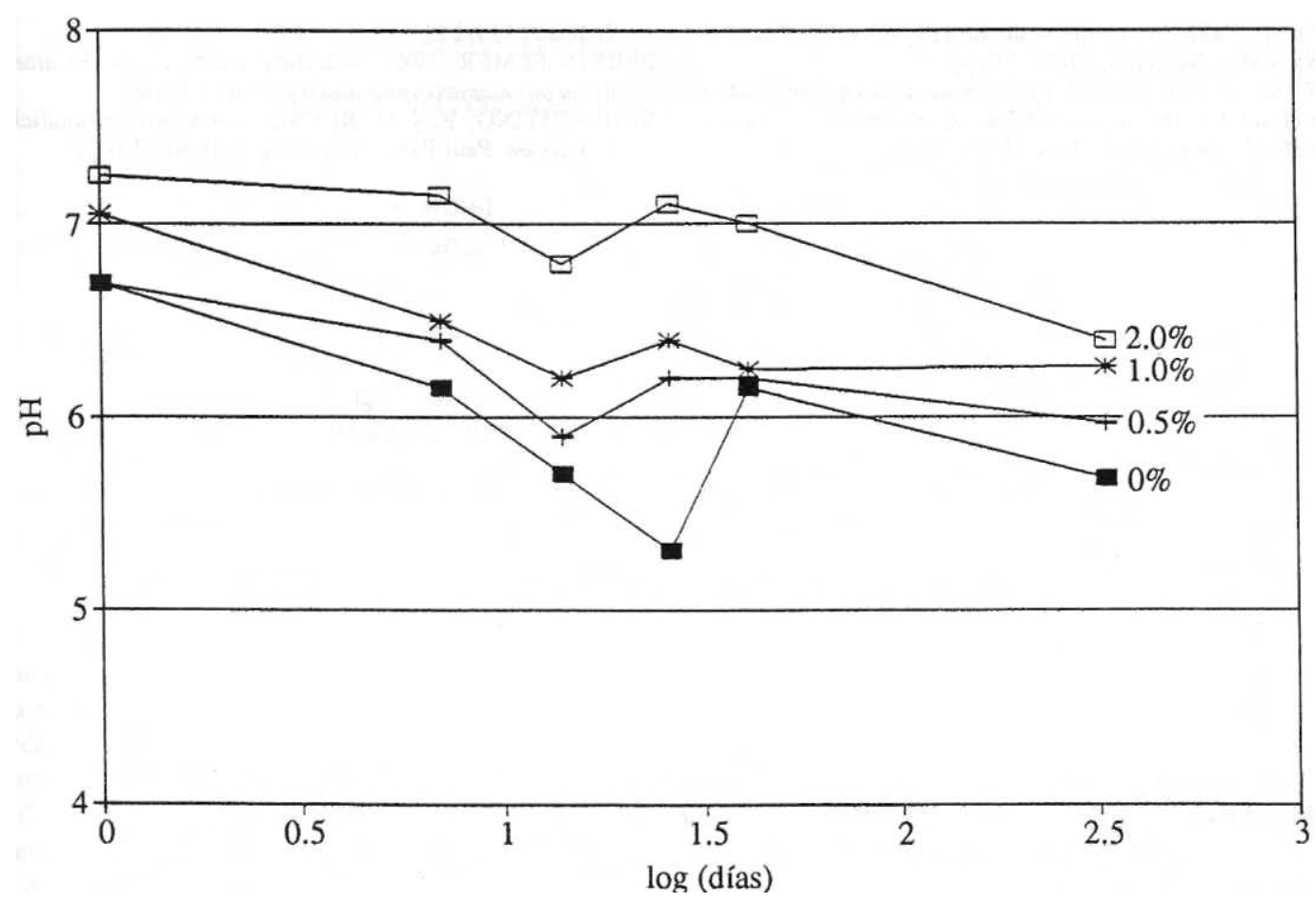

Fig. 1. Variación del pH en el tiempo para mezclas de suelos y trumao (c) con ceniza en distintas dosis. $\mathrm{pH}$ variation over time for mixtures of trumao soils with wood ash of distinct doses.

El efecto de la ceniza CEFOR en el pH de los suelos (cuadro 3) muestra un claro aumento de éste, alcanzándose el rango comprendido entre $\mathrm{pH}$ 6 y 7 con dosis de ceniza del orden de $0.5,1.0$ y $2.0 \%$.

El valor de $\mathrm{pH}$ de las mezclas suelo-ceniza contenidas en los maceteros se muestra en la figura 1. Estos maceteros fueron mantenidos a la intemperie entre mayo de 1989 y abril de 1990, recibiendo los efectos climáticos directamente, en particular una pluviosidad de $1.589 \mathrm{~mm}$.

Se puede concluir que en todos los suelos se logró un incremento del valor original de $\mathrm{pH}$ en rangos dependientes de las dosis de ceniza adicionada y, fundamentalmente, que tal efecto se mantuvo en el tiempo, no obstante la elevada pluviosidad recibida. A sí, para el suelo rojo arcilloso, transcurrido prácticamente un año bajo las condiciones antes señaladas, el suelo sin ceniza presenta un $\mathrm{pH}$ 5.97, la dosis $1.0 \%$ un pH 6.40 y la dosis $2.0 \%$ un pH 6.71. Para el suelo trumao los valores respectivos son: $5.68,5.97,6.30$ y 6.40 y para el suelo pardo metamórfico de 5.61,5.98,6.15 y 6.47 .

En este sentido, y coincidiendo con las conclusiones logradas por Naylor y Schmidt (1986), los resultados permiten inferir que una aplicación práctica de este residuo sería su utilización como enmienda alcalina, favoreciendo una elevación del $\mathrm{pH}$ en suelos ácidos.

\section{BIBLIOGRAFIA}

ADAMS, F. 1984. Crop response to Lime in the Southern U nited States. En: Soil Acidity and Liming. 2nd. ed., ASA, CSSA, SSSA. Ser, A gronomy $\mathrm{N}^{\circ} 12$.

BOLT, G. y M. BRUGGENWERT. 1978. Soil Chemistry, Part A: Basic Elements. Elsevier Scientific Publishing Company, $281 \mathrm{pp}$.

CHILE FORESTAL. 1987a. "A mpliación y modernización de Celulosa A rauco", Chile Forestal 138: 10-12. 1987b. "A provechamiento energético de residuos forestales", Chile Forestal 138: 25-26.

1987c. "Demanda por leña: ¿un problema inquietante?", Chile Forestal 138: 23-26. 
FINCK, A. 1979. Dünger und Düngung. Verlag Chemie.

IREN-CORFO-UACH. 1978. Estudio de suelos de la provincia de Valdivia. Instituto de Investigación de Recursos N aturales, Santiago, Chile, $178 \mathrm{pp}$.

MURPHY, J. y J.P. RILEY. 1962. "A modified single solution method for the determination of phosphate in natural waters". Anal. Chim. Acta 27: 31-36.
NAY LOR, L.M. y E.J. SCHMIDT. 1986. "A gricultural use of a wood ash as a fertilizer and liming material", TAPPI Journal: 114-119.

PERKIN ELMER. 1982. Analytical methods for the atomic absorption spectrophotometry. Perkin Elmer.

SCHLICHTING, E. y H. BLUME. 1966. Bodenkundliches praklikum. Paul Parey, Hamburg y Berlin, 209 pp. 